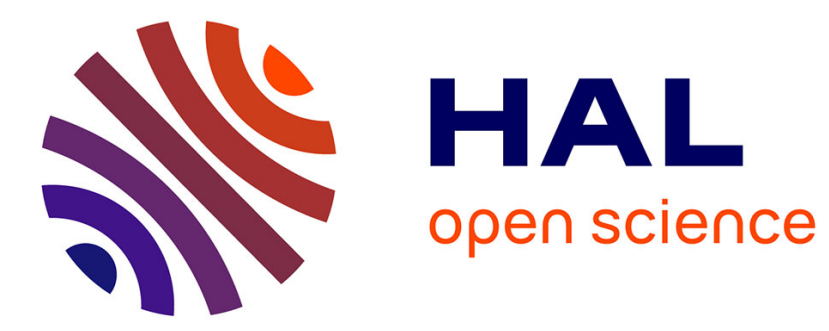

\title{
Spherical wave reflection in layered media with rough interfaces: Three-dimensional modeling
}

Samuel Pinson, Julio Cordioli, Laurent Guillon

\section{To cite this version:}

Samuel Pinson, Julio Cordioli, Laurent Guillon. Spherical wave reflection in layered media with rough interfaces: Three-dimensional modeling. Journal of the Acoustical Society of America, 2016, 140 (2), pp.1108-1115. 10.1121/1.4961000 . hal-01397195

\section{HAL Id: hal-01397195 \\ https://hal.science/hal-01397195}

Submitted on 15 Nov 2016

HAL is a multi-disciplinary open access archive for the deposit and dissemination of scientific research documents, whether they are published or not. The documents may come from teaching and research institutions in France or abroad, or from public or private research centers.
L'archive ouverte pluridisciplinaire HAL, est destinée au dépôt et à la diffusion de documents scientifiques de niveau recherche, publiés ou non, émanant des établissements d'enseignement et de recherche français ou étrangers, des laboratoires publics ou privés. 


\title{
Spherical wave reflection in layered media with rough interfaces: Three-dimensional modeling
}

\author{
Samuel Pinson ${ }^{\text {a) }}$ and Julio Cordioli \\ Laboratório de Vibração e Acústica, Universidade Federal de Santa Catarina, Floriaónopolis, Brazil \\ Laurent Guillon \\ Institut de Recherche de l'Ecole Navale, BCRM Brest, CC600, 29240 Brest Cedex 9, France
}

(Received 21 October 2015; revised 29 June 2016; accepted 28 July 2016; published online 17 August 2016)

\begin{abstract}
In the context of sediment characterization, layer interface roughnesses may be responsible for sound-speed profile measurement uncertainties. To study the roughness influence, a threedimensional (3D) modeling of a layered seafloor with rough interfaces is necessary. Although roughness scattering has an abundant literature, $3 \mathrm{D}$ modeling of spherical wave reflection on rough interfaces is generally limited to a single interface (using Kirchhoff-Helmholtz integral) or computationally expensive techniques (finite difference or finite element method). In this work, it is demonstrated that the wave reflection over a layered medium with irregular interfaces can be modeled as a sum of integrals over each interface. The main approximations of the method are the tangentplane approximation, the Born approximation (multiple reflection between interfaces are neglected) and flat-interface approximation for the transmitted waves into the sediment. The integration over layer interfaces results in a method with reasonable computation cost. (C) 2016 Acoustical Society of America. [http://dx.doi.org/10.1121/1.4961000]
\end{abstract}

Pages: $1108-1115$

\section{INTRODUCTION}

In the context of sediment characterization, the seafloor is generally assumed to be made of a stack of layers with flat interfaces. A recently developed method for sediment soundspeed profile characterization is the image source method (ISM). ${ }^{1}$ This technique is based on the analysis of the seafloor reflected acoustic wave as a collection of image sources whose positions are linked with the thicknesses and the sound speed of the sediment stack. In Ref. 1, the ISM is applied to experimental data acquired by the NATO Undersea Research Center in 2009 during the Clutter'09 experiment. The equipment consisted of an autonomous undersea vehicle towing a $1600-3500 \mathrm{~Hz}$ frequency band source and a $32 \mathrm{~m}$ horizontal line array of 32 hydrophones at $12 \mathrm{~m}$ above the seabed. Under the assumption of locally range independent seabed properties, the moving horizontal array provided successive range independent sediment sound-speed profiles along a track to obtain the range and depth dependent structure of the seafloor. From this study, it was observed that the sound-speed profiles obtained by the ISM are subject to important instabilities from one measurement to another. It is suspected that interface roughnesses might play an important role in those instabilities. To study roughness influence on sound-speed profile measurement it is necessary to simulate wave reflection on a layered medium with rough interfaces so that every parameter can be controlled carefully. Also, the three-dimensional (3D) effect in interface roughness scattering has to be taken into account.

Although roughness scattering has an abundant literature, most of the existing models for wave reflection in layered media with rough interfaces are statistical and used to evaluate the

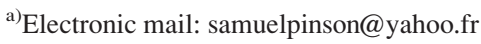

scattering strength (or scattering cross section). ${ }^{2-7}$ To simulate a deterministic signal, 3D modeling of spherical wave reflection on rough interfaces is generally limited to a single interface (using the Kirchhoff approximation of the Kirchhoff-Helmholtz integral) 8,9 or computationally expensive (using the finitedifference method). ${ }^{10}$ For wave propagation in range dependent layered media, ray theory can be used for wave propagation through the medium and combined with the Kirchhoff approximation for the interface reflection modeling. ${ }^{11}$ In this work, a similar approach is adopted. The method uses three approximations: the tangent-plane approximation, the Born approximation (multiple reflections between interfaces are neglected), and flatinterface approximation for the transmitted waves.

In Sec. II, the environmental configuration for the synthetic data is first described before detailing the 3D data modeling in Sec. III. Finally in Sec. IV the results obtained from simulated signals are presented.

\section{ENVIRONMENTAL CONFIGURATION}

The configuration of the numerical experiment consists of a layered medium modeled as a three layer stack with geoacoustic parameters defined in Table I. A semi-infinite water layer is considered so that the sea-surface reflections are not included in the model. In the method described in Sec. III, the model geometry is defined by the layer's interfaces. Their mean planes are parallel, $120 \mathrm{~m}$ wide and $150 \mathrm{~m}$ long (Fig. 1). The spatial sampling used to define the roughness height is set to $2.5 \mathrm{~cm}$.

Interface roughnesses between layers are generated such that the spatial power spectrum has a "von Karman" spectrum form ${ }^{12}$

$$
W_{2}\left(k_{x}, k_{y}\right)=\frac{w_{2}}{\left(k_{x}^{2}+k_{y}^{2}+1 / L^{2}\right)^{\gamma_{2} / 2}},
$$


where $k_{x}$ and $k_{y}$ are the horizontal wavenumbers, $2 \pi L$ is the roughness correlation length, $\gamma_{2}$ the spectral exponent, and $w_{2}$ is the spectral strength. Values for $L$ and $\gamma_{2}$ are fixed, respectively, to $10 \mathrm{~m}$ and 3 , and the spectral strength is set to $w_{2}=3.18 \times 10^{-4}$. With these parameters, the root mean square $(\mathrm{rms})$ roughness height is $\zeta_{\mathrm{rms}}=14 \mathrm{~cm}$. The $\mathrm{rms}$ roughness height $\zeta_{\mathrm{rms}}$ is given by

$$
\zeta_{\mathrm{rms}}^{2}=\frac{2 \pi w_{2}}{\left(\gamma_{2}-2\right) L^{-\left(\gamma_{2}-2\right)}}
$$

The rms roughness height is smaller than the wavelength at the central frequency $(1.5 \mathrm{~m}$ in water) but important enough to play a significant role on interface wave reflections.

The source and the receiver are $20 \mathrm{~m}$ apart and $10 \mathrm{~m}$ above the first interface. The source signal has a Gaussian envelope with a $1 \mathrm{kHz}$ central frequency and a bandwidth of $600 \mathrm{~Hz}$. Thus, the pulse duration corresponds to a $4 \mathrm{~m}$ length in water that provides a layer thickness resolution of about $2 \mathrm{~m}$. In the time domain, the emitted pulse maximum amplitude is set to 1 at $1 \mathrm{~m}$ from the source. The sampling frequency is set to $12 \mathrm{kHz}$.

\section{THREE-DIMENSIONAL MODELING OF SPHERICAL WAVE REFLECTION IN LAYERED MEDIA WITH ROUGH INTERFACES}

\section{A. Wave equation in inhomogeneous media}

In the inhomogeneous medium presented in Fig. 2, the harmonic acoustic pressure $P(\mathbf{r})$ at coordinate $\mathbf{r}=(x, y, z)$ and frequency $\omega$ obeys the wave equation

$$
\rho(\mathbf{r}) \nabla \frac{1}{\rho(\mathbf{r})} \nabla P(\mathbf{r})+\left(\frac{\omega}{c(\mathbf{r})}\right)^{2} P(\mathbf{r})=-S \delta\left(\mathbf{r}-\mathbf{r}_{A}\right),
$$

where $S$ is the source amplitude at frequency $\omega$ and $\mathbf{r}_{A} \in \mathcal{D}_{0}$ is the source coordinate. The inhomogeneous medium is composed of the homogeneous domains $\mathcal{D}_{0}$ to $\mathcal{D}_{L}$ separated by the interfaces $S_{1}$ to $S_{L}$ (Fig. 2).

The density $\rho(\mathbf{r})$ and the sound speed $c(\mathbf{r})$ are constant inside the layers, i.e., $\rho(\mathbf{r})=\rho_{l}$ and $c(\mathbf{r})=c_{l}$ for $\mathbf{r} \in \mathcal{D}_{l}$. As in Refs. 5 and 13, a Green function is defined for each volume $\mathcal{D}_{l}$,

$$
\Delta G_{l}\left(\mathbf{r}^{\prime}, \mathbf{r}\right)+\left(\frac{\omega}{c_{l}}\right)^{2} G_{l}\left(\mathbf{r}^{\prime}, \mathbf{r}\right)=-\delta\left(\mathbf{r}^{\prime}-\mathbf{r}\right),
$$

for which the 3D solution is the Green's function for the infinite, homogeneous medium,

TABLE I. Layered media parameters.

\begin{tabular}{lccc}
\hline \hline \multicolumn{2}{l}{ Layer thickness Sound speed $(\mathrm{m} / \mathrm{s})$} & Density $\left(\mathrm{kg} / \mathrm{m}^{3}\right)$ & Absorption $(\mathrm{dB} / \mathrm{m} / \mathrm{kHz})$ \\
\hline Water & 1500 & 1000 & 0 \\
$3 \mathrm{~m}$ & 1490 & 1100 & 0.1 \\
$7 \mathrm{~m}$ & 1550 & 1300 & 0.5 \\
$10 \mathrm{~m}$ & 1600 & 1500 & 0.5 \\
Basement & 1700 & 1700 & 0.3 \\
\hline
\end{tabular}

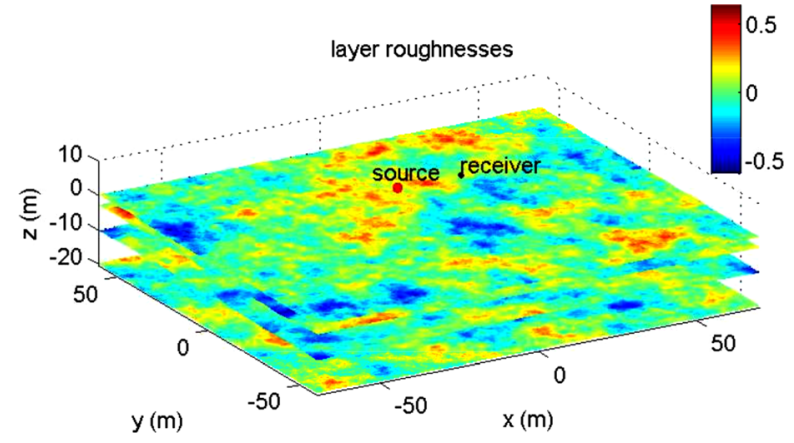

FIG. 1. (Color online) Example of a roughness realization for the four interfaces. The color scale corresponds to the roughness height relative to the interface mean plane (in $\mathrm{cm}$ ).

$$
G_{l}\left(\mathbf{r}^{\prime}, \mathbf{r}\right)=\frac{\mathrm{e}^{\mathrm{i} k_{l}\left|\mathbf{r}^{\prime}-\mathbf{r}\right|}}{4 \pi\left|\mathbf{r}^{\prime}-\mathbf{r}\right|}
$$

where $\mathbf{r}, \mathbf{r}^{\prime} \in \mathcal{D}_{l}$ and $k_{l}=\omega / c_{l}$.

Then Eq. (3) and Eq. (4) are multiplied by $G_{l}\left(\mathbf{r}^{\prime}, \mathbf{r}\right)$ and $P(\mathbf{r})$, respectively, subtracted, integrated over the volume $\mathcal{D}_{l}$, and the Green's theorem is applied. This results in the set of integral equations as follows:

$$
\begin{aligned}
P\left(\mathbf{r}^{\prime}\right)= & S G_{0}\left(\mathbf{r}^{\prime}, \mathbf{r}_{0}\right)+\int_{S_{1}}\left[G_{0}\left(\mathbf{r}^{\prime}, \mathbf{r}\right) \nabla_{\mathbf{n}} P(\mathbf{r})\right. \\
& \left.-P(\mathbf{r}) \nabla_{\mathbf{n}} G_{0}\left(\mathbf{r}^{\prime}, \mathbf{r}\right)\right] \mathrm{d} \mathbf{r},
\end{aligned}
$$

for $\mathbf{r}^{\prime} \in \mathcal{D}_{0}$ and

$$
P\left(\mathbf{r}^{\prime}\right)=\int_{S_{l} \cup S_{l+1}}\left[G_{l}\left(\mathbf{r}^{\prime}, \mathbf{r}\right) \nabla_{\mathbf{n}} P(\mathbf{r})-P(\mathbf{r}) \nabla_{\mathbf{n}} G_{l}\left(\mathbf{r}^{\prime}, \mathbf{r}\right)\right] \mathrm{d} \mathbf{r}
$$

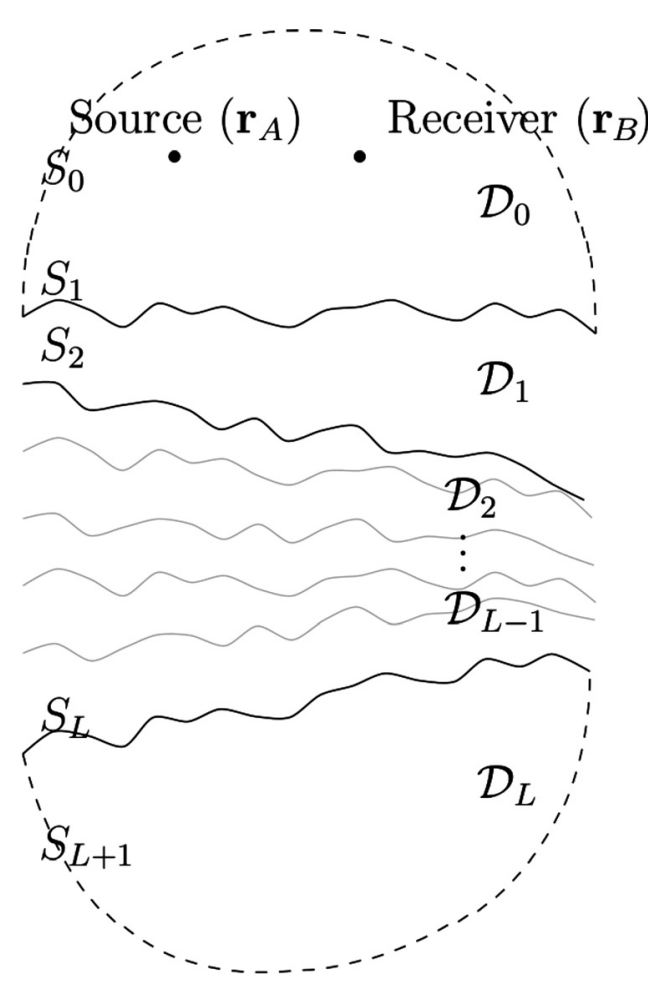

FIG. 2. Geometry of the problem. 
for $\mathbf{r}^{\prime} \in \mathcal{D}_{l}, l \neq 0, \mathrm{~d} \mathbf{r}$ is the surface element, $\nabla_{\mathbf{n}}$ is the normal derivative on the interface, and $\mathbf{n}=\left(n_{x}, n_{y}, n_{z}\right)$ is the normal vector to the interface at point $\mathbf{r}$.

Integrals over $S_{0}, S_{L+1}$, and the layer sides vanish because of the free field Sommerfeld radiation condition.

\section{B. Kirchhoff and Born approximation in layered media}

In a configuration with a single interface sufficiently smooth and if the incident wave from the source can be considered as locally plane, one can use the Kirchhoff approximation (also called tangent plane approximation). ${ }^{15}$ Thus, the pressure on the interface is $P(\mathbf{r}) \approx P^{i}(\mathbf{r})+P^{r}(\mathbf{r})$ where $P^{i}(\mathbf{r})$ is the incident wave from the source and $P^{r}(\mathbf{r})$ is the locally reflected wave. To account for the scattered wave from below the interface, the approximation for $P(\mathbf{r})$ in Eq. (6) is

$$
P_{0}(\mathbf{r}) \approx P_{0}^{i}(\mathbf{r})+P_{0}^{r}(\mathbf{r})+P_{0}^{t}(\mathbf{r})
$$

where the subscript 0 in $P_{0}(\mathbf{r})$ has been added to refer to the volume $\mathcal{D}_{0}$ in which the pressure is evaluated, $P_{0}^{r}(\mathbf{r})$ $=R_{01} P_{0}^{i}(\mathbf{r})$ is the reflected pressure in $\mathcal{D}_{0}, R_{01}$ is the local reflection coefficient from $\mathcal{D}_{0}$ on the interface $S_{1}$, and $P_{0}^{t}(\mathbf{r})=T_{10} P_{1}^{s}(\mathbf{r})$ is the transmitted wave through the interface $S_{1} . P_{1}^{s}(\mathbf{r})$ is the scattered field below the interface $S_{1}$, and $T_{10}$ is the transmission coefficient from $\mathcal{D}_{1}$ to $\mathcal{D}_{0}$. To be strict, the scattered field below the interface $P_{1}^{s}(\mathbf{r})$ should be decomposed into a sum of locally plane waves, each one having its own transmission coefficient. Nevertheless, the term $T_{10} P_{1}^{s}(\mathbf{r})$ is used for the sake of clarity. Then, the pressure and its normal derivative in Eq. (6) are

$$
\begin{aligned}
P_{0}\left(\mathbf{r}_{S 1}\right) \approx & P_{0}^{i}\left(\mathbf{r}_{S 1}\right)+R_{01} P_{0}^{i}\left(\mathbf{r}_{S 1}\right)+T_{10} P_{1}^{s}\left(\mathbf{r}_{S 1}\right) \\
\nabla_{\mathbf{n}} P\left(\mathbf{r}_{S 1}\right) \approx & \nabla_{\mathbf{n}} P_{0}^{i}\left(\mathbf{r}_{S 1}\right)-R_{01} \nabla_{\mathbf{n}} P_{0}^{i}\left(\mathbf{r}_{S 1}\right) \\
& +T_{10} \nabla_{\mathbf{n}} P_{1}^{s}\left(\mathbf{r}_{S 1}\right)
\end{aligned}
$$

Using this approximation, Eq. (6) for a receiver at the coordinate $\mathbf{r}_{B}$ becomes

$$
P_{0}\left(\mathbf{r}_{B}\right)=4 \pi S G_{0}\left(\mathbf{r}_{B}, \mathbf{r}_{A}\right)+\mathcal{I}\left(\mathbf{r}_{B}\right)+\mathcal{I}_{S 1}\left(\mathbf{r}_{B}\right)+\mathcal{I}_{S 1}^{S}\left(\mathbf{r}_{B}\right),
$$

with

$$
\begin{aligned}
\mathcal{I}\left(\mathbf{r}_{B}\right)= & \int_{S_{1}}\left[G_{0}\left(\mathbf{r}_{B}, \mathbf{r}_{S 1}\right) \nabla_{\mathbf{n}} P_{0}^{i}\left(\mathbf{r}_{S 1}\right)\right. \\
& \left.-P_{0}^{i}\left(\mathbf{r}_{S 1}\right) \nabla_{\mathbf{n}} G_{0}\left(\mathbf{r}_{B}, \mathbf{r}_{S 1}\right)\right] \mathrm{d} \mathbf{r}_{S 1}=0, \\
\mathcal{I}_{S 1}\left(\mathbf{r}_{B}\right)= & -\int_{S_{1}}\left[G_{0}\left(\mathbf{r}_{B}, \mathbf{r}_{S 1}\right) R_{01} \nabla_{\mathbf{n}} P_{0}^{i}\left(\mathbf{r}_{S 1}\right)\right. \\
& \left.+R_{01} P_{0}^{i}\left(\mathbf{r}_{S 1}\right) \nabla_{\mathbf{n}} G_{0}\left(\mathbf{r}_{B}, \mathbf{r}_{S 1}\right)\right] \mathrm{d} \mathbf{r}_{S 1}, \\
\mathcal{I}_{S 1}^{S}\left(\mathbf{r}_{B}\right)= & \int_{S_{1}}\left[G_{0}\left(\mathbf{r}_{B}, \mathbf{r}_{S 1}\right) T_{10} \nabla_{\mathbf{n}} P_{1}^{S}\left(\mathbf{r}_{S 1}\right)\right. \\
& \left.-T_{10} P_{1}^{s}\left(\mathbf{r}_{S 1}\right) \nabla_{\mathbf{n}} G_{0}\left(\mathbf{r}_{B}, \mathbf{r}_{S 1}\right)\right] \mathrm{d} \mathbf{r}_{S 1} .
\end{aligned}
$$

The integral $\mathcal{I}\left(\mathbf{r}_{B}\right)$ [Eq. (11)] is null because it corresponds to the wave field solution in absence of the reflector.
The integral $\mathcal{I}_{S 1}\left(\mathbf{r}_{B}\right)$ is known as the Kirchhoff approximation for the reflected wave from the interface $S 1$ and the integral $\mathcal{I}_{S 1}^{s}\left(\mathbf{r}_{B}\right)$ represents the scattered field from below the interface $S_{1}$ and is a function of the pressure $P_{1}^{s}(\mathbf{r})$ in $\mathcal{D}_{1}$.

If the reflection on $S_{1}$ of the up-going wave inside $\mathcal{D}_{1}$ is neglected, one can approximate Eq. (7) for the scattered pressure in $\mathcal{D}_{1}$ by

$$
\begin{aligned}
P_{1}^{s}\left(\mathbf{r}_{S 1}\right)= & \int_{S_{2}}\left[G_{1}\left(\mathbf{r}_{S 1}, \mathbf{r}_{S 2}\right) \nabla_{\mathbf{n}} P_{1}\left(\mathbf{r}_{S 2}\right)\right. \\
& \left.-P_{1}\left(\mathbf{r}_{S 2}\right) \nabla_{\mathbf{n}} G_{1}\left(\mathbf{r}_{S 1}, \mathbf{r}_{S 2}\right)\right] \mathrm{d} \mathbf{r}_{S 2},
\end{aligned}
$$

where $\mathbf{r}_{S 1} \in S_{1}$ and $\mathbf{r}_{S 2} \in S_{2}$.

Neglecting the up-going wave reflections on the upper interface inside the layers is, in other words, neglecting the multiple reflections inside the layers. For that reason this approximation is referred as the Born approximation.

As for interface $S_{1}$ the approximation $P_{1}\left(\mathbf{r}_{S 2}\right)$ $\approx P_{1}^{i}\left(\mathbf{r}_{S 2}\right)+P_{1}^{r}\left(\mathbf{r}_{S 2}\right)+P_{1}^{t}\left(\mathbf{r}_{S 2}\right)$ on $S_{2}$ is used. Thus the pressure $P_{1}^{s}$ in $\mathcal{D}_{1}$ is

$$
\begin{aligned}
P_{1}^{s}\left(\mathbf{r}_{S 1}\right)= & -\int_{S_{2}}\left[G_{1}\left(\mathbf{r}_{S 1}, \mathbf{r}_{S 2}\right) R_{12} \nabla_{\mathbf{n}} P_{1}^{i}\left(\mathbf{r}_{S 2}\right)\right. \\
& \left.+R_{12} P_{1}^{i}\left(\mathbf{r}_{S 2}\right) \nabla_{\mathbf{n}} G_{1}\left(\mathbf{r}_{S 1}, \mathbf{r}_{S 2}\right)\right] \mathrm{d} \mathbf{r}_{S 2} \\
& +\int_{S_{2}}\left[G_{1}\left(\mathbf{r}_{S 1}, \mathbf{r}_{S 2}\right) T_{21} \nabla_{\mathbf{n}} P_{2}^{s}\left(\mathbf{r}_{S 2}\right)\right. \\
& \left.-T_{21} P_{2}^{s}\left(\mathbf{r}_{S 2}\right) \nabla_{\mathbf{n}} G_{1}\left(\mathbf{r}_{S 1}, \mathbf{r}_{S 2}\right)\right] \mathrm{d} \mathbf{r}_{S 2},
\end{aligned}
$$

where $P_{2}^{s}\left(\mathbf{r}_{S 2}\right)$ is the scattered field from below the interface $S 2$. Now, one can replace $P_{1}^{s}\left(\mathbf{r}_{S 1}\right)$ in $\mathcal{I}_{S 1}^{s}\left(\mathbf{r}_{B}\right)$ by its expression in Eq. (16). After rearranging the terms inside the integrals over $S_{1}$ and $S_{2}$, one can write $\mathcal{I}_{S 1}^{s}\left(\mathbf{r}_{B}\right)$ in the following form:

$$
\mathcal{I}_{S 1}^{s}\left(\mathbf{r}_{B}\right)=\mathcal{I}_{S 2}\left(\mathbf{r}_{B}\right)+\mathcal{I}_{S 2}^{s}\left(\mathbf{r}_{B}\right)
$$

with

$$
\begin{aligned}
\mathcal{I}_{S 2}\left(\mathbf{r}_{B}\right)= & -\int_{S_{2}}\left[\mathcal{G}_{1 \rightarrow 0}\left(\mathbf{r}_{B}, \mathbf{r}_{S 2}\right) R_{12} \nabla_{\mathbf{n}} P_{1}^{i}\left(\mathbf{r}_{S 2}\right)\right. \\
& \left.+R_{12} P_{1}^{i}\left(\mathbf{r}_{S 2}\right) \nabla_{\mathbf{n}} \mathcal{G}_{1 \rightarrow 0}\left(\mathbf{r}_{B}, \mathbf{r}_{S 2}\right)\right] \mathrm{d} \mathbf{r}_{S 2},
\end{aligned}
$$

$$
\begin{aligned}
\mathcal{I}_{S 2}^{S}\left(\mathbf{r}_{B}\right)= & \int_{S_{2}}\left[\mathcal{G}_{1 \rightarrow 0}\left(\mathbf{r}_{B}, \mathbf{r}_{S 2}\right) T_{21} \nabla_{\mathbf{n}} P_{2}^{S}\left(\mathbf{r}_{S 2}\right)\right. \\
& \left.-T_{21} P_{2}^{S}\left(\mathbf{r}_{S 2}\right) \nabla_{\mathbf{n}} \mathcal{G}_{1 \rightarrow 0}\left(\mathbf{r}_{B}, \mathbf{r}_{S 2}\right)\right] \mathrm{d} \mathbf{r}_{S 2},
\end{aligned}
$$

where

$$
\begin{aligned}
\mathcal{G}_{1 \rightarrow 0}\left(\mathbf{r}_{B}, \mathbf{r}_{S 2}\right)= & \int_{S_{1}}\left[G_{0}\left(\mathbf{r}_{B}, \mathbf{r}_{S 1}\right) T_{10} \nabla_{\mathbf{n}} G_{1}\left(\mathbf{r}_{S 1}, \mathbf{r}_{S 2}\right)\right. \\
& \left.-T_{10} G_{1}\left(\mathbf{r}_{S 1}, \mathbf{r}_{S 2}\right) \nabla_{\mathbf{n}} G_{0}\left(\mathbf{r}_{S 1}, \mathbf{r}_{S 2}\right)\right] \mathrm{d} \mathbf{r}_{S 1}
\end{aligned}
$$

is defined as the Green's function from $\mathcal{D}_{1}$ to $\mathcal{D}_{0}$. Similarly, the incident pressure in $\mathcal{D}_{1}$ is given by

$$
P_{1}^{i}\left(\mathbf{r}_{S 2}\right)=4 \pi S \mathcal{G}_{0 \rightarrow 1}\left(\mathbf{r}_{S 2}, \mathbf{r}_{A}\right)
$$


where

$$
\begin{aligned}
\mathcal{G}_{0 \rightarrow 1}\left(\mathbf{r}_{S 2}, \mathbf{r}_{A}\right)= & \int_{S_{1}}\left[G_{1}\left(\mathbf{r}_{S 2}, \mathbf{r}_{S 1}\right) T_{01} \nabla_{\mathbf{n}} G_{0}\left(\mathbf{r}_{S 1}, \mathbf{r}_{A}\right)\right. \\
& \left.-T_{01} G_{0}\left(\mathbf{r}_{S 1}, \mathbf{r}_{A}\right) \nabla_{\mathbf{n}} G_{1}\left(\mathbf{r}_{S 2}, \mathbf{r}_{S 1}\right)\right] \mathrm{d} \mathbf{r}_{S 1} .
\end{aligned}
$$

Using the same reasoning for all interfaces, the recorded pressure at the coordinate $\mathbf{r}_{B}$ can be modeled as a sum of integrals over each interface,

$$
P_{0}\left(\mathbf{r}_{B}\right)=4 \pi S G_{0}\left(\mathbf{r}_{B}, \mathbf{r}_{A}\right)+\sum_{l=1}^{L} \mathcal{I}_{S l}\left(\mathbf{r}_{B}\right),
$$

with

$$
\begin{aligned}
\mathcal{I}_{S l}\left(\mathbf{r}_{B}\right)= & -4 \pi S \int_{S_{l}}\left[\mathcal{G}_{l-1 \rightarrow 0}\left(\mathbf{r}_{B}, \mathbf{r}_{S l}\right)\right. \\
& \times R_{l-1 l} \nabla_{\mathbf{n}} \mathcal{G}_{0 \rightarrow l-1}\left(\mathbf{r}_{S l}, \mathbf{r}_{A}\right) \\
& +R_{l-1 l} \mathcal{G}_{0 \rightarrow l-1}\left(\mathbf{r}_{S l}, \mathbf{r}_{A}\right) \\
& \left.\times \nabla_{\mathbf{n}} \mathcal{G}_{1-1 \rightarrow 0}\left(\mathbf{r}_{B}, \mathbf{r}_{S l}\right)\right] \mathrm{d} \mathbf{r}_{S l},
\end{aligned}
$$

where the Green's function between a point $\mathbf{r} \in \mathcal{D}_{q}$ and the receiver point $\mathbf{r}_{B} \in \mathcal{D}_{0}$ for $q \geq 1$ is obtained by the recurrence relation

$$
\begin{aligned}
\mathcal{G}_{q \rightarrow 0}\left(\mathbf{r}_{B}, \mathbf{r}\right)= & \int_{S_{q}}\left[\mathcal{G}_{q-1 \rightarrow 0}\left(\mathbf{r}_{B}, \mathbf{r}_{S q}\right) T_{q q-1} \nabla_{\mathbf{n}} G_{q}\left(\mathbf{r}, \mathbf{r}_{S q}\right)\right. \\
& \left.-T_{q q-1} G_{q}\left(\mathbf{r}, \mathbf{r}_{S q}\right) \nabla_{\mathbf{n}} \mathcal{G}_{q-1 \rightarrow 0}\left(\mathbf{r}_{B}, \mathbf{r}_{S q}\right)\right] \mathrm{d} \mathbf{r}_{S q},
\end{aligned}
$$

as well as the Green's function between the source point $\mathbf{r}_{A} \in \mathcal{D}_{0}$ and the point $\mathbf{r} \in \mathcal{D}_{q}$,

$$
\begin{aligned}
\mathcal{G}_{0 \rightarrow q}\left(\mathbf{r}, \mathbf{r}_{A}\right)= & \int_{S_{q}}\left[G_{q}\left(\mathbf{r}, \mathbf{r}_{S q}\right) T_{q-1 q} \nabla_{\mathbf{n}} \mathcal{G}_{0 \rightarrow q-1}\left(\mathbf{r}_{S q}, \mathbf{r}_{A}\right)\right. \\
& \left.-T_{q-1 q} \mathcal{G}_{0 \rightarrow q-1}\left(\mathbf{r}_{S q}, \mathbf{r}_{A}\right) \nabla_{\mathbf{n}} G_{q}\left(\mathbf{r}, \mathbf{r}_{S q}\right)\right] \mathrm{d} \mathbf{r}_{S q} .
\end{aligned}
$$

Equation (23) is an important intermediary result. Starting from the wave equation in inhomogeneous media, under the tangent-plane approximation and neglecting the multiple reflections inside a layer (Kirchhoff and Born approximation), it has been shown that the reflected wave from a layered medium can be understood as a sum of the reflections from each interface.

\section{Ray approximation}

Equations (20) and (22) for $\mathcal{G}_{1 \rightarrow 0}\left(\mathbf{r}_{A}, \mathbf{r}_{S 2}\right)$ and $\mathcal{G}_{0 \rightarrow 1}\left(\mathbf{r}_{S 2}, \mathbf{r}_{A}\right)$ can be recognized as the Kirchhoff approximation for transmitted waves between two fluids separated by a moderately rough interface. ${ }^{9,17}$ Rewriting Eq. (22) using the high frequency approximation, we obtain

$$
\begin{aligned}
\mathcal{G}_{0 \rightarrow 1}\left(\mathbf{r}_{S 2}, \mathbf{r}_{A}\right) \approx & \int_{S_{1}} \mathrm{i} \omega T_{01} \frac{\left(\mathbf{n} \cdot \mathbf{u}_{0} / c_{0}+\mathbf{n} \cdot \mathbf{u}_{1} / c_{1}\right)}{4 \pi R_{0} 4 \pi R_{1}} \\
& \times \mathrm{e}^{\mathrm{i} \omega\left(\tau_{0}+\tau_{1}\right)} \mathrm{d} \mathbf{r}_{S 1},
\end{aligned}
$$

where "." is the scalar product, $\tau_{0}=\left|\mathbf{r}_{S 1}-\mathbf{r}_{A}\right| / c_{0}$ and $\tau_{1}$ $=\left|\mathbf{r}_{S 2}-\mathbf{r}_{S 1}\right| / c_{1}$ are the travel times in $\mathcal{D}_{0}$ and $\mathcal{D}_{1}, \mathbf{u}_{0}$ $=\left(\mathbf{r}_{S 1}-\mathbf{r}_{A}\right) /\left|\mathbf{r}_{S 1}-\mathbf{r}_{A}\right|$ and $\mathbf{u}_{1}=\left(\mathbf{r}_{S 2}-\mathbf{r}_{S 1}\right) /\left|\mathbf{r}_{S 2}-\mathbf{r}_{S 1}\right|$ are the unit vector of incidence in $\mathcal{D}_{0}$ and $\mathcal{D}_{1}, R_{0}=\left|\mathbf{r}_{S 1}-\mathbf{r}_{A}\right|$ and $R_{1}=\left|\mathbf{r}_{S 2}-\mathbf{r}_{S 1}\right|$.

The transmitted wave through a moderately rough interface is mainly affected by the roughness at high angle of incidence (or low grazing angle). ${ }^{9,17}$ Otherwise, one can approximate the integral in Eq. (27) with the flat interface corresponding to the mean plane $\bar{S}_{1}$. Then, the stationary phase evaluation of the integral gives ${ }^{16}$

$$
\mathcal{G}_{0 \rightarrow 1}\left(\mathbf{r}_{S 2}, \mathbf{r}_{A}\right) \approx \tilde{T}_{01} \frac{1}{4 \pi} \frac{\left(\nabla_{\mathbf{n}} \tilde{\tau}_{0}-\nabla_{\mathbf{n}} \tilde{\tau}_{1}\right)}{\tilde{R}_{0} \tilde{R}_{1}} \frac{1}{2 \sqrt{|H|}} \mathrm{e}^{\mathrm{i} \omega\left(\tilde{\tau}_{0}+\tilde{\tau}_{1}\right)},
$$

where the tilde over the variables stands for their values at the stationary phase path, $|H|=\left|\nabla_{S 1} \cdot\left(\nabla_{S 1}\left(\tilde{\tau}_{0}+\tilde{\tau}_{1}\right)\right)^{\mathbf{T}}\right|$ is the determinant of the Hessian matrix and $\nabla_{S 1}$ is the gradient in the interface $\bar{S}_{1}$.

This result may be difficult to generalize in the recurrence relation of Eqs. (25) and (26) for an arbitrary number of interfaces. So the ray path of stationary phase will be considered and the ray amplitudes and delays are calculated using the ray theory. Considering that the wave is transmitted through the flat mean planes $\bar{S}_{l}$, the function $\mathcal{G}_{0 \rightarrow q}\left(\mathbf{r}, \mathbf{r}_{A}\right)$ [Eqs. (26) and (25)] between a point $\mathbf{r} \in \mathcal{D}_{q}$ and the point $\mathbf{r}_{A} \in \mathcal{D}_{0}$ are calculated using Langston's method. ${ }^{18}$ It consists of multiplying the geometric divergence of the ray, ${ }^{19}$ the product of the transmission coefficient through each interface and the phase term

$$
\mathcal{G}_{0 \rightarrow q}\left(\mathbf{r}, \mathbf{r}_{A}\right) \approx A\left(\mathbf{r}, \mathbf{r}_{A}\right) \mathrm{e}^{\mathrm{i} \omega \tau^{\star}}
$$

with

$$
A\left(\mathbf{r}, \mathbf{r}_{A}\right) \approx\left|\frac{c_{q} J\left(\mathbf{r}_{A}\right)}{c_{0} J(\mathbf{r})}\right|^{1 / 2} \times \prod_{p=1}^{q} T_{p-1 p},
$$

where $J\left(\mathbf{r}_{A}\right)=1$ and the Jacobian of the ray is ${ }^{19}$

$$
J(\mathbf{r})=\left|\begin{array}{lll}
\frac{\partial x}{\partial s} & \frac{\partial x}{\partial \theta_{0}} & \frac{\partial x}{\partial \psi_{0}} \\
\frac{\partial y}{\partial s} & \frac{\partial y}{\partial \theta_{0}} & \frac{\partial y}{\partial \psi_{0}} \\
\frac{\partial z}{\partial s} & \frac{\partial z}{\partial \theta_{0}} & \frac{\partial z}{\partial \psi_{0}}
\end{array}\right|,
$$

$\theta_{0}$ and $\psi_{0}$ are the ray incidence angle and the ray azimuth at the origin $\mathbf{r}_{A}$, respectively.

To include absorption, the complex sound speed $c_{l}^{\star}$ $=c_{l} /\left(1+\mathrm{i} \beta_{l}\right)$ is used (the symbol ${ }^{\star}$ indicates a complex valued parameter). The parameter $\beta_{l}$ can be obtained from the absorption coefficient $\alpha_{l}$ in $\mathrm{dB} / \mathrm{m} / \mathrm{kHz}$ by ${ }^{20}$

$$
\beta_{l}=\alpha_{l} \times \frac{c_{l}}{1000(2 \pi) 20 \log _{10} \mathrm{e}} .
$$

Thus, the travel time $\tau^{\star}$ in Eq. (29) is complex and can be calculated from the eikonal equation, 


$$
\begin{aligned}
\tau^{\star} & =\int \frac{1}{c^{\star}(s)} \mathrm{d} s \\
& =\int \frac{1}{c(s)} \mathrm{d} s+\mathrm{i} \int \frac{\beta(s)}{c(s)} \mathrm{d} s,
\end{aligned}
$$

where $s$ is the coordinate along the ray path between $\mathbf{r}$ and $\mathbf{r}_{A}$.

Integrals in Eq. (23) for the scattered field can then be simplified using the high frequency approximation,

$$
\nabla_{\mathbf{n}} \mathcal{G}_{0 \rightarrow q}\left(\mathbf{r}^{\prime}, \mathbf{r}\right) \approx \mathrm{i} k_{q} \mathbf{u}_{q} \cdot \mathbf{n} \mathcal{G}_{0 \rightarrow q}\left(\mathbf{r}^{\prime}, \mathbf{r}\right)
$$

where $\mathbf{u}_{q}$ is the incident unit vector in $\mathcal{D}_{q}$.

Applying this approximation in Eq. (24) and using Eq. (29), we obtain

$$
\begin{aligned}
\mathcal{I}_{S l}\left(\mathbf{r}_{B}\right) \approx & -4 \pi S \int_{S_{l}} R_{l-1 l}\left(\mathrm{i} k_{l-1} \mathbf{u}^{i} \cdot \mathbf{n}+\mathrm{i} k_{l-1} \mathbf{u}^{r} \cdot \mathbf{n}\right) \\
& \times A\left(\mathbf{r}_{S l}, \mathbf{r}_{A}\right) A\left(\mathbf{r}_{B}, \mathbf{r}_{S l}\right) \mathrm{e}^{\mathrm{i} \omega\left(\tau^{i}+\tau^{r}\right)} \mathrm{d} \mathbf{r}_{S l},
\end{aligned}
$$

where $\mathbf{u}^{i}$ and $\mathbf{u}^{r}$ are the unit vector of the ray from $\mathbf{r}_{A}$ and $\mathbf{r}_{B}$ on $\mathbf{r}_{S l}, \tau^{i}$ is the travel time between $\mathbf{r}_{A}$ and $\mathbf{r}_{S l}$, and $\tau^{r}$ is the travel time between $\mathbf{r}_{S l}$ and $\mathbf{r}_{B}$ (superscript ${ }^{i}$ and ${ }^{r}$ stand for incident and reflected, respectively).

By convention, vectors $\mathbf{u}$ and $\mathbf{n}$ are pointing downward and upward, respectively, in Eq. (38). When $\mathbf{u}^{i} \cdot \mathbf{n}>0$ or $\mathbf{u}^{r} \cdot \mathbf{n}>0$, the surface element $\mathrm{d} \mathbf{r}_{S l}$ is in a shadow. The amplitudes for those shadowed elements is set to 0 to improve the range of validity of the Kirchhoff approximation. ${ }^{21}$

The roughness height $\zeta$ and its normal on every point of the surface are a known input of the problem. As a small roughness is assumed, the incident unit vector on the rough interface $\mathbf{u}$ is almost equal to the incident unit vector $\overline{\mathbf{u}}$ on the mean plane $\overline{S_{l}}$ (Fig. 3). Thus, we can also approximate the complex travel time by

$$
\tau^{\star}=\overline{\tau^{\star}}-\zeta u_{z} / c_{l-1},
$$

where $\overline{\tau^{\star}}$ is the complex travel time to the mean plane $\overline{S_{l}}$.

One can show that the surface element $\mathrm{d} \mathbf{r}_{S l}=\mathrm{d} \overline{\mathbf{r}_{S l}} / n_{z}$ where $\mathrm{d} \overline{\mathbf{r}_{S l}}$ is the surface element of the mean plane. Eventually, if we neglect the roughness in the geometric divergence in $A$, Eq. (35) can be written by

$$
\begin{aligned}
\mathcal{I}_{S l}\left(\mathbf{r}_{B}\right) \approx & -4 \pi S \int_{\overline{S_{l}}} R_{l-1 l} A\left(\overline{\mathbf{r}_{S l}}, \mathbf{r}_{A}\right) A\left(\mathbf{r}_{B}, \overline{\mathbf{r}_{S l}}\right) \\
& \times\left(\mathrm{i} k_{l-1} \overline{\mathbf{u}^{i}} \cdot \mathbf{n}+\mathrm{i} k_{l-1} \overline{\mathbf{u}^{r}} \cdot \mathbf{n}\right) \\
& \times \exp \left\{\mathrm{i} \omega\left(\overline{\tau^{i \star}}+\overline{\tau^{r \star}}-\frac{\zeta u_{z}^{i}+\zeta u_{z}^{r}}{c_{l-1}}\right)\right\} \frac{\mathrm{d} \overline{\mathbf{r}_{S l}}}{n_{z}},
\end{aligned}
$$

where the plane wave reflection and transmission coefficient are given by ${ }^{14}$

$$
\begin{aligned}
R_{l-1 l} & =\frac{m \cos \theta-\sqrt{n^{2}-\sin ^{2} \theta}}{m \cos \theta+\sqrt{n^{2}-\sin ^{2} \theta}}, \\
T_{p-1 p} & =\frac{2 m \cos \theta}{m \cos \theta+\sqrt{n^{2}-\sin ^{2} \theta}},
\end{aligned}
$$

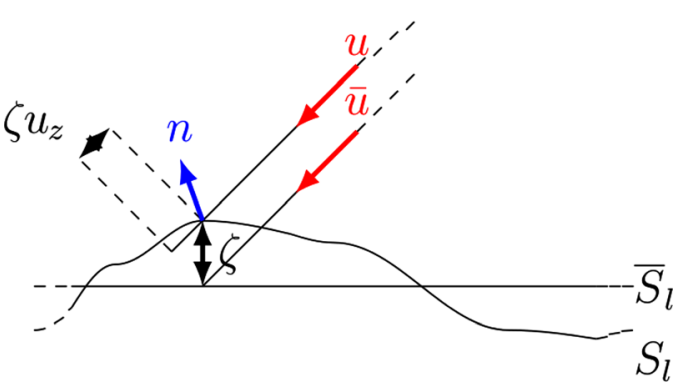

FIG. 3. (Color online) Zoom on the ray intersection with the rough interface and its mean plane.

with $m=\rho_{l} / \rho_{l-1}, n=c_{l-1}^{\star} / c_{l}^{\star}$ and $\cos \theta=\left|\mathbf{u}_{l-1} \cdot \mathbf{n}\right|$ is the cosine of the incidence angle above the interface. For the transmission coefficient, the normal $\mathbf{n}$ is taken from the mean plane.

The expression for $A\left(\mathbf{r}_{B}, \overline{\mathbf{r}}\right)$ is similar to Eq. (30) with the upgoing wave transmission coefficient $T_{p p-1}$ instead of $T_{p-1 p}$.

\section{Numerical evaluation}

Equation (37) has a convenient form for numerical evaluation. One can calculate the ray paths, geometric divergence and transmission coefficient through previous interfaces from the source and the receiver to the mean plane, and apply a correction factor in the travel times to include the roughness. All these quantities are frequency independent so that they can be calculated before the integration at a given frequency. Then, integration is performed for each frequency component of the signal to be simulated and the time-domain simulated signal at coordinate $\mathbf{r}_{B}$ is obtained using the inverse Fourier transform,

$$
p_{0}\left(\mathbf{r}_{B}, t\right)=\frac{1}{2 \pi} \int P_{0}\left(\mathbf{r}_{B}, \omega\right) \mathrm{e}^{\mathrm{i} \omega t} \mathrm{~d} \omega .
$$

To calculate the ray paths, the method described by Langston ${ }^{18}$ consists of the following steps:

(1) Send a ray parameterized by the angles $\theta_{0}$ and $\psi_{0}$ from the source and receiver location.

(2) Calculate its intersection coordinate on next interface.

(3) Calculate the transmission coefficient and the Snell's ray refraction.

(4) Iterate 2 and 3 to the last interface.

(5) Calculate reflection coefficient $R_{l-1 l}$ for the ray coming from the source $\mathbf{r}_{A}$.

For a given ray with parameters $\left(\theta_{0}, \psi_{0}\right)$, two other rays have to be sent with parameters $\left(\theta_{0}+\delta \theta, \psi_{0}\right)$ and $\left(\theta_{0}, \psi_{0}\right.$ $+\delta \psi)$ in order to evaluate numerically the derivatives in the Jacobian [Eq. (31)].

Note that, using Langston's method, interface mean planes do not have to be parallel to each other.

At this step an issue needing to be addressed remains. For a given ray parameterized by the angles $\theta_{0}$ and $\psi_{0}$, it is difficult to define where the ray intersects the interface for which the roughness is predefined on a regular mesh. Figure 4 illustrates the issue. The lines represent many rays sent 


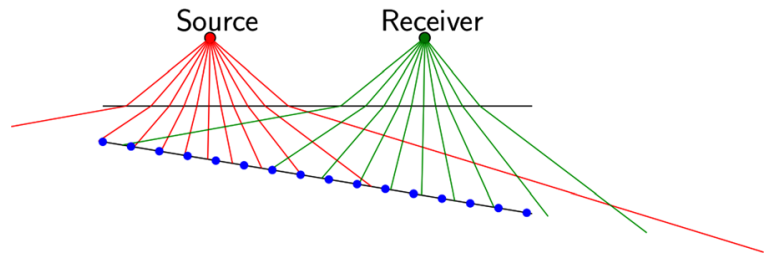

FIG. 4. (Color online) Rays sent from the source and the receiver to the interface. Dots represent the regular mesh for which the roughness has been generated.

from the source or receiver to the interface, and the dots represent the regular mesh for which the roughness has been generated.

This issue is solved by noticing that quantities related to the mean plane in Eq. (37) vary smoothly. So one can send many rays to the mean plane and then interpolate all the ray parameters (travel time to the mean plane, incidence unit vector, transmission coefficients and geometric divergence) in Eq. (37) on the predefined roughness mesh. Then, $\zeta$ and $\mathbf{n}$ being known, one can apply the travel time correction $\zeta u_{z} / c_{l-1}$ due to the roughness and calculate the local reflection coefficient.

Figure 5 illustrates the interpolation for the ray geometric divergence from the receiver on the fourth interface of the configuration described in Sec. II. In this case, rays are sent to cover the predefined mean plane with a $50 \mathrm{~cm}$ sample length in $x$ and $y$. Ray parameters $\theta_{0}$ and $\psi_{0}$ are calculated to reach that grid as if there were no ray refraction. Of course, due to ray refraction, rays do not reach those points and leak from the grid but it is sufficient to cover the predefined roughness grid and interpolate the values (the boundary of the roughness mesh is displayed by the black rectangle in Fig. 5).

\section{RESULTS}

\section{A. Flat interface case}

First, a signal is simulated with flat interfaces and the layered medium properties described in Sec. II [Fig. 6(a)]. One can see the four reflections of the four interfaces and a weak

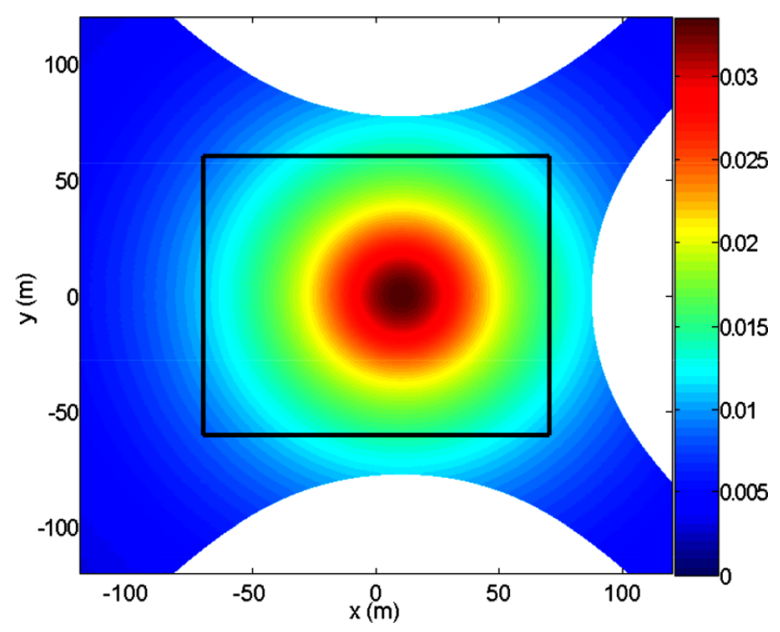

FIG. 5. (Color online) Geometric divergence of rays sent from the receiver to cover the fourth interface mean plane of the configuration described in Sec. II. The black rectangle represents the boundary of the predefined roughness grid where ray parameters have to be interpolated. (a)

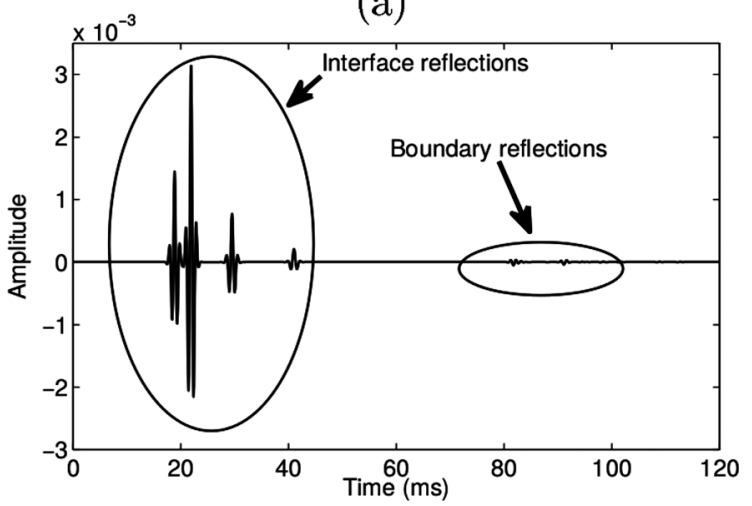

(b)

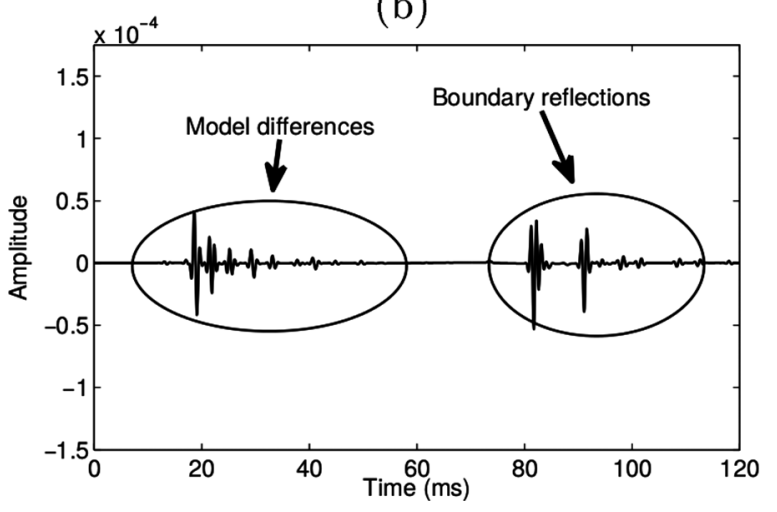

FIG. 6. (a) Simulated signal with flat interfaces. (b) Difference between the simulated signal and the numerical evaluation of the Sommerfeld integral (the vertical scale is exaggerated by a factor of 20 ).

backscattering after $t=80 \mathrm{~ms}$. This backscattering comes from the diffraction at the interface boundaries. For comparison, another signal is calculated by a numerical evaluation of the Sommerfeld integral, the exact analytical solution of the reflection of a spherical wave in layered media, 22,23

$$
\begin{aligned}
P_{0}^{r}\left(\mathbf{r}_{B}, \mathbf{r}_{A}, \omega\right)= & \mathrm{i} k \int_{0}^{\pi / 2-\mathrm{i} \infty} \mathrm{J}_{0}\left(\left\|r_{B}-r_{A}\right\| k \sin \theta\right) R(\theta, \omega) \\
& \times \mathrm{e}^{\mathrm{i} k\left(z_{A}+z_{B}\right) \cos \theta} \sin \theta \mathrm{d} \theta,
\end{aligned}
$$

where $r=\sqrt{x^{2}+y^{2}}, \theta$ is the angle of incidence, $k$ the wave number and $\mathrm{J}_{0}$ is the zeroth order Bessel function of the first kind. Because this integral is the result of plane wave decomposition, the term $R(\theta, \omega)$ is the plane wave reflection coefficient and can be computed for an arbitrary layering of fluid or elastic media. ${ }^{14}$

The simulated signals from the two models are almost equal so the analysis is done on their difference in Fig. 6(b) [the vertical scale is exaggerated by a factor of 20 compared to Fig. 6(a)]. The small differences between the two models are mainly explained by the presence of multiple reflections present in the simulation from the Sommerfeld integral simulation. Also it appears that interface echoes themselves (at times $t=19,22,30$, and $41 \mathrm{~ms}$ ) present some small differences. Note that multiple reflections are very weak in amplitude. Indeed, the order of magnitude of the reflection coefficients from one layer to another is $\mathcal{O}(0.1)$ so the order of magnitude of the first multiple reflection is $\mathcal{O}\left(10^{-3}\right)$, i.e., $\approx 40 \mathrm{~dB}$ lower than direct echoes. 


\section{B. Two-dimensional case}

Signals obtained by a two dimensional version of the method with rough interfaces are compared to data computed by specfem $2 \mathrm{D},{ }^{24}$ a spectral element code developed to simulate propagation of seismic waves at the Earth scale. ${ }^{25}$ This software has been applied to underwater acoustics problems ${ }^{26}$ and to T-wave generation and propagation modeling. ${ }^{27}$ Absorption is not included in those simulations. Interfaces are $140 \mathrm{~m}$ long with a $10 \mathrm{~cm}$ spatial sampling.

The first 2D simulation is performed with a sufficiently smooth roughness compatible with the Kirchhoff approximation. For that purpose, a Gaussian spatial power spectrum is used to generate the rough interfaces,

$$
W_{1}\left(k_{x}\right)=\frac{L \zeta_{\text {rms }}^{2}}{2 \sqrt{\pi}} \mathrm{e}^{-k_{x}^{2} L^{2} / 4}
$$

with a correlation length $L=10 \mathrm{~m}$ and a rms roughness height $\zeta_{\mathrm{rms}}=14 \mathrm{~cm}$.

The 2D simulated signal is plotted in Fig. 7(a). The direct path between source and receiver has been included in this simulation (echo at $t=13 \mathrm{~ms}$ ). The result fits well with the specfem2D simulation, the difference between the two models is plotted in Fig. 7(b) [the vertical scale is exaggerated by a factor of 10 compared to Fig. 7(a)]. Interface echo differences (at times $t=19,22,30$, and $41 \mathrm{~ms}$ ) are comparable in amplitude with the multiple reflections between interfaces.

A second 2D simulation is performed with a roughness having a von Karman spatial power spectrum for a better

(a)

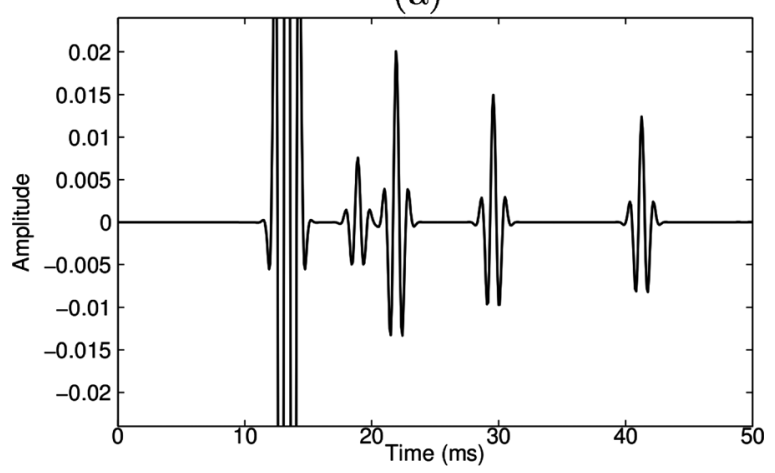

(b)

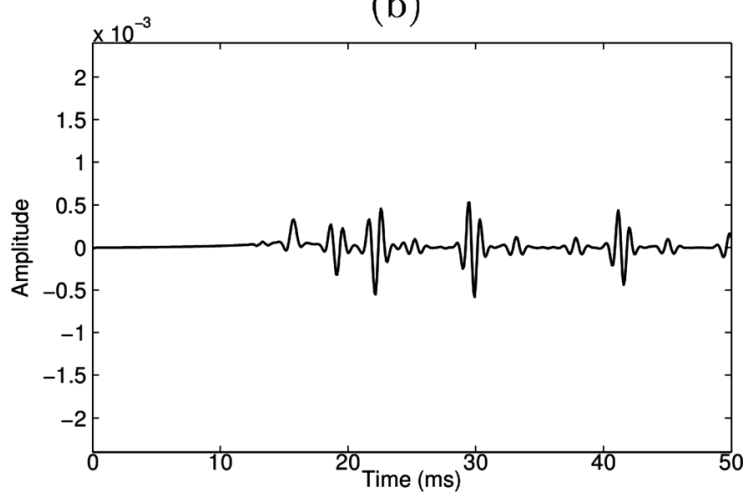

FIG. 7. (a) $2 \mathrm{D}$ simulated signal with rough interfaces having a Gaussian power spectrum. (b) Difference between the simulated signal and the result computed by specfem $2 \mathrm{D}$ (the vertical scale is exaggerated by a factor of 10 ). (a)

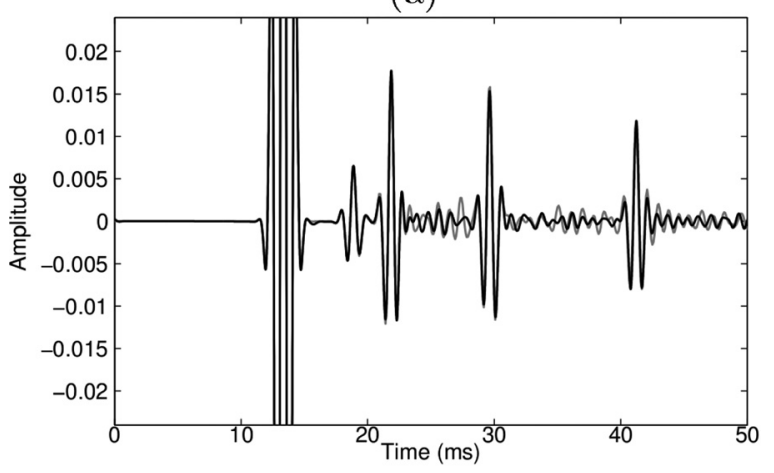

(b)

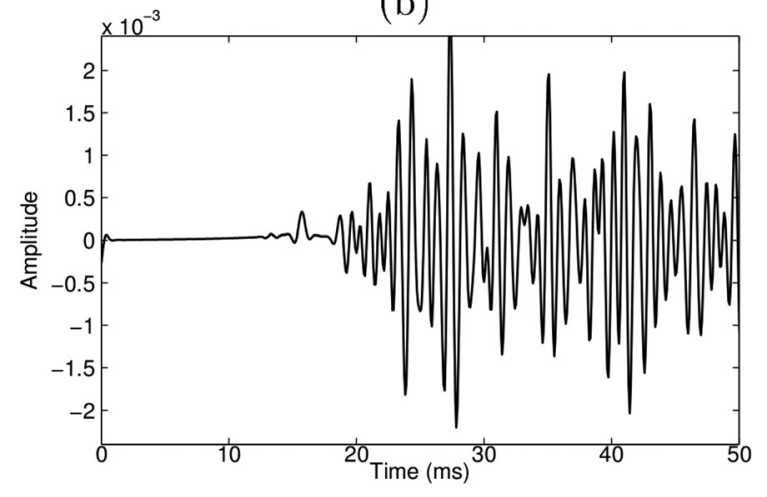

FIG. 8. (a) Gray curve: 2D simulated signal with rough interfaces having a von Karman power spectrum. Black curve: data computed by specfem2D. (b) Difference between the simulated signal and the result computed by specfem2D (the vertical scale is exaggerated by a factor of 10 ).

understanding of the model behavior in the presence of high spatial frequency components. The von Karman spectrum is given by ${ }^{12}$

$$
W_{1}\left(k_{x}\right)=\frac{w_{1}}{\left(k_{x}^{2}+1 / L^{2}\right)^{\gamma_{1} / 2}},
$$

where $\gamma_{1}$ is the spectral exponent, and $w_{1}$ is the spectral strength. Values for $L$ and $\gamma_{1}$ are fixed to $10 \mathrm{~m}$ and 2, respectively, and the spectral strength is set to $w_{1}=6.36 \times 10^{-4}$. With these parameters, the roughness is equivalent to the $3 \mathrm{D}$ case presented in Sec. II.

The simulated signal is plotted in gray in Fig. 8(a) and the specfem2D simulation is plotted in black. Interface echoes fit well in amplitude and travel times, but visible differences appear between and after these echoes. Those differences reveal the limitations of the Kirchhoff approximation for the modeling of the incoherent part of the signal. Model errors are more clear in Fig. 8(b) where the difference between the two models is plotted (vertical scale exaggerated by a factor of 10). Nevertheless, the differences are not significant when the interest is in the specular reflections. Moreover the computation cost is about a thousand time lower.

\section{Three-dimensional case}

The results from simulations with the rough interfaces described in Sec. II are shown in Fig. 9 for two different realizations of the roughness. One can identify three interface echoes and the fourth interface echo is hidden in the 


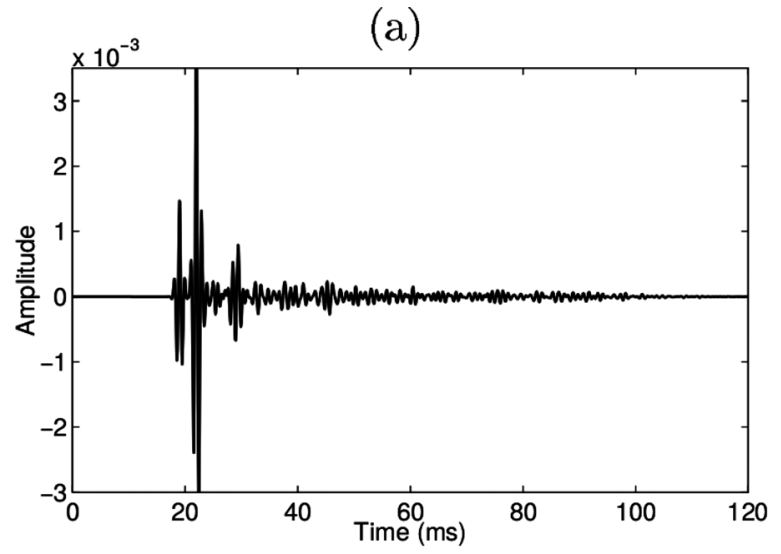

(b)

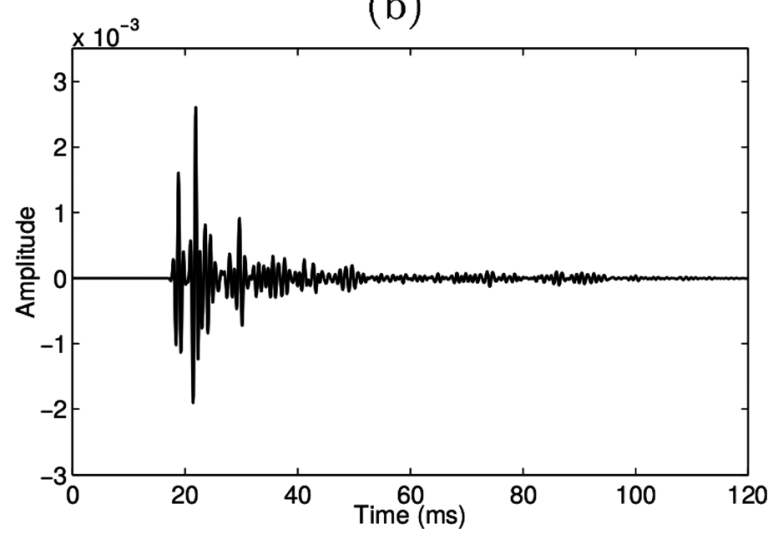

FIG. 9. (a) and (b) 3D simulated signals for two different realizations of the roughness.

roughness backscattering. Also, echo amplitudes are affected by the roughness. The cause of these amplitude variations is probably a focusing/defocusing effect from large scale roughness oscillations. Moreover, one can see the incoherent backscattering between and after the interface echoes. From the $2 \mathrm{D}$ case, it is reasonable to be confident in echo amplitudes and arrival times but the incoherent backscattering is likely to be over estimated.

\section{CONCLUSION}

A 3D modeling of spherical wave reflection in layered media with rough interfaces has been developed. The method uses three approximations: the tangent-plane approximation, the Born approximation (multiple reflections between interfaces are neglected) and the flat-interface approximation for the transmitted waves. Also, it has been shown that a wave reflection on a layered medium with rough interfaces can be written as a sum of integrals over each interface. In future work, this model will be used to study the roughness influence in sediment sound-speed profile measurement when using a source and an array of hydrophones.

\section{ACKNOWLEDGMENTS}

This work is supported by the Coordenação de Aperfeiçoamento de Pessoal de Nível Superior from Brazil through the young talent funding and partially funded by Wavetech company. The authors would like to thank Derek Olson for his help on the rough surface simulation.
${ }^{1}$ S. Pinson, L. Guillon, and C. Holland, "Range dependent sediment sound speed profile measurements using the image source method," J. Acoust. Soc. Am. 134, 156-165 (2013).

${ }^{2}$ L. Guillon and X. Lurton, "Backscattering from buried sediment layers: The equivalent input backscattering strength model," J. Acoust. Soc. Am. 109, 122-132 (2001).

${ }^{3}$ D. R. Jackson, R. I. Odom, M. L. Boyd, and A. N. Ivakin, “A geoacoustic bottom interaction model (GABIM)," IEEE J. Ocean. Eng. 35, 603-617 (2010).

${ }^{4} \mathrm{C}$. Wu and X. Zhang, "Second-order perturbative solutions for 3-D electromagnetic radiation and propagation in a layered structure with multilayer rough interfaces,” IEEE J. Selected Topics Appl. Earth Obs. Remote Sensing 8, 180-194 (2015).

${ }^{5}$ J.-Y. Liu, P.-C. Hsueh, and C.-F. Huang, "Coherent reflection of acoustic plane wave from a rough seabed, with a random sediment layer overlying an elastic basement," IEEE J. Ocean. Eng. 27, 853-861 (2002).

${ }^{6}$ A. N. Ivakin, "A unified approach to volume and roughness scattering," J. Acoust Soc. Am. 103, 827-837 (1998).

${ }^{7}$ A. N. Ivakin, "Models for seafloor roughness and volume scattering," in OCEANS' 98 Conference Proceedings (1998), Vol. 1, pp. 518-521.

${ }^{8}$ E. Pouliquen, A. P. Lyons, and N. G. Pace, "The Helmholtz-Kirchhoff approach to modeling penetration of acoustic waves into rough seabeds," J. Acoust. Soc. Am. 104, 1762-1762 (1998).

${ }^{9}$ E. Pouliquen, A. Lyons, and N. G. Pace, "Penetration of acoustic waves into rippled sandy seafloors," J. Acoust. Soc. Am. 108, 2071-2081 (2000).

${ }^{10}$ W. Makinde, N. Favretto-Cristini, and E. De Bazelaire, "Numerical modeling of interface scattering of seismic wavefield from a random rough interface in an acoustic medium: Comparison between 2D and 3D cases," Geophys. Prospecting 53, 373-397 (2005).

${ }^{11}$ V. Cerveny, Seismic Ray Theory (Cambridge University Press, Cambridge, UK, 2001), pp. 417-449.

${ }^{12}$ D. Jackson and M. Richardson, High-frequency Seafloor Acoustics (Springer, New York, 2007), pp. 475-479.

${ }^{13}$ D. Tang and B. T. Hefner, "Modeling interface roughness scattering in a layered seabed for normal-incident chirp sonar signals," J. Acoust Soc. Am. 131, EL302-EL308 (2012).

${ }^{14}$ L. Brekhovskikh and Y. Lysanov, "Reflection of sound from the surface and bottom of the ocean: plane waves," in Fundamentals of Ocean Acoustics (Springer-Verlag, Berlin, 1991), Chap. 3, pp. 61-79.

${ }^{15} \mathrm{~L}$. Brekhovskikh and Y. Lysanov, "Scattering of sound at rough surfaces," in Fundamentals of Ocean Acoustics (Springer-Verlag, Berlin, 1991), Chap. 9, pp. 183-226.

${ }^{16}$ N. Bleistein, Mathematical Methods for Wave Phenomena (Academic Press, Orlando, FL, 1984), pp. 82-88.

${ }^{17} \mathrm{~T}$. Lieuwen, "Explicit results for wave scattering and transmission through a rough fluid-fluid interface," Appl. Acoust. 63, 1031-1050 (2002).

${ }^{18}$ C. A. Langston, "The effect of planar dipping structure on source and receiver responses for constant ray parameter," Bull. Seismol. Soc. Am. 67, 1029-1050 (1977).

${ }^{19}$ F. Jensen, W. Kupperman, M. Porter, and H. Shmidt, "Ray methods," in Computational Ocean Acoustics (AIP, New York, 1994), pp. 155-232.

${ }^{20}$ F. Jensen, W. Kupperman, M. Porter, and H. Shmidt, "Fundamentals of ocean acoustics," in Computational Ocean Acoustics (AIP, New York, 1994), pp. 1-64.

${ }^{21}$ E. I. Thorsos, "The validity of the Kirchhoff approximation for rough surface scattering using a Gaussian roughness spectrum," J. Acoust. Soc. Am. 83, 78-92 (1988).

${ }^{22}$ L. Brekhovskikh and O. Godin, Acoustics of Layered Media. II: Point Sources and Bounded Beams (Springer-Verlag, Berlin, 1999), pp. 1-15.

${ }^{23}$ C. W. Holland, P. Nielsen, J. Dettmer, and S. Dosso, "Resolving meso-scale seabed variability using reflection measurements from an autonomous underwater vehicle," J. Acoust. Soc. Am. 131, 1066-1078 (2012).

${ }^{24}$ Specfem $2 D$ User Manual, version 7.0, available at http://geodynamics.org/ cig/software/specfem2d/ (Last viewed June 29, 2016).

${ }^{25}$ D. Komatitsch and J. Vilotte, "The spectral element method: An efficient tool to simulate the seismic response on 2D and 3D geological structure," Bull. Seismol. Soc. Am. 88, 368-392 (1998).

${ }^{26} \mathrm{P}$. Cristini and D. Komatitsch, "Some illustrative examples of the use of a spectral-element method in ocean acoustics," J. Acoust. Soc. Am. 131, EL229-EL235 (2012).

${ }^{27}$ G. Jamet, C. Guennou, L. Guillon, C. Mazoyer, and J. Y. Royer, "T-wave generation and propagation: A comparison between data and spectral element modeling," J. Acoust. Soc. Am. 134, 3376-3385 (2013). 